\title{
Erythropoietin preconditioning on hippocampus neuronal apoptosis following status epilepticus induced by Li-pilocarpine in rats through anti-caspase-3 expression
}

\author{
XiaoNi Wen, YuanGui Huang, JinCun Wang \\ Department of Neurology, Xijing Hospital, The Fourth Military Medical University of Chinese PLA, China
}

\begin{abstract}
Selective neuronal loss following status epilepticus(SE) was first described just under 100 years ago. The acute pathology following SE was shown to be 'ischemic cell change' and was assumed to arise through hypoxia/ ischemia. Recently, erythropoietin (Epo) has been shown to have potent anti-apoptosis activity in central nervous system neurons in animal models of ischaemic injury. Aims: In this report, in order to determine Epo preconditioning on hippocampus neuronal apoptosis, we examined caspase3 expression following SE caused by Li-pilocarpine in rats. Settings and Design: Animals were classified into three groups: EP group (pilocarpine group), rhEpo-pilocarpine group and control group. Four hours after preconditioning with Epo intraperitoneally, pilocarpine hydrochloride was administered intraperitoneally and observed for behavioral manifestations of SE. The animals were sacrificed at one hour after SE onset. Materials and Methods: At the abovementioned time point, animals were deeply anesthetized and were perfused through the left ventricle. Detection of hippocampus neuronal apoptosis was performed with caspase-3 immunohistochemical technique on three groups. To further confirm which cell population upregulates caspase-3, brain sections were stained for NeuN (green) and caspase-3 (red).Statistical analysis ANOVA and Fisher's post hoc test was used. Results Quatification of hippocampus neurons revealed that the number of caspase-3-positive cells in the CA1/CA3 area and dentate gyrus(DG) of three groups had a significant difference. In comparison with control group, there was an increase by $74 \%$ and $534 \%, 42 \%$ and $272 \%$ in the CA $1 / C A 3$ area and DG of EP group and rhEpo-treated group respectively. There was a decrease by $18 \%$ and $26 \%$ in the CA1/CA3 area and DG of rhEpo-treated group compared with those in EP group. In addition, colocalization of caspase-3 with NeuN was shown. Conclusions Systemic rhEpo therapy reduced caspase-3 expression in SE induced by Li-pilocarpine.
\end{abstract}

Key words: Status epilepticus (SE), erythropoietin (Epo), apoptosis, hippocampus, pretreatment.
Status epilepticus (SE) is a neurological emergency with an associated mortality rate of 10-12\%. Pilocarpine-induced seizure models have provided information on the behavioral and neurochemical characteristics associated with seizure activity. This model can be used to investigate the development of neuropathology in SE. ${ }^{[1,12]}$ Selective neuronal loss following SE was first described just under 100 years ago. The acute pathology following SE was shown to be 'ischemic cell change' and was assumed to arise through hypoxia/ischemia. ${ }^{[2]}$

As erythropoietin (Epo) has been to be neuroprotective in the central nervous system (CNS) ${ }^{[3]}$, we hypothesized that exogenous Epo therapy would be of value in treating hypoxia/ischemia nerve injury by reducing neuron apoptosis following SE. In this report, we examined the extent of apoptosis in hippocampus. This was carried out in the context of experimental Epo preconditioning, where we systemically delivered recombinant human Epo (rhEpo) four hours before pilocarpine injection and identified changes in hippocampus neurons and seizure activity during SE.

In addition, as we know, caspase-3, a member of the family of cysteine proteases, mediates neuronal apoptosis that is induced following trophic factor withdrawal, exposures to inhibitors of protein kinases or phosphatases, or hypoxic-ischemic brain injury. ${ }^{[4]}$ Selective neuronal loss following SE was assumed to arise through hypoxia/ischemia. Experimental modeling of such brain injury suggests that seizures elicit a mixture of necrotic and apoptotic cell death within vulnerable fields. Features suggestive of apoptosis following seizures include altered expression of BCL2 -family proteins, activation of caspases, and neuroprotective effects of caspase inhibitors. ${ }^{[5]}$

Therefore, in the present study, activation of caspase-3 was shown to mediate hippocampus neuronal apoptosis. We provide evidence that erythropoietin reduced hippocampus neuronal apoptosis through inhibiting activation of caspase-3. 


\section{Materials and Methods}

\section{Animal treatments}

Young adult male Sprague-Dawley rats (180-200g) were housed under a 12:12 h light/dark cycle with free access to food and water. Four hours after preconditioning with Epo (10U/g) intraperitoneally ${ }^{[6]}$ thirty mg per kilogram body weight pilocarpine hydrochloride (Sigma, St. Louis, MO) was administered intraperitoneally following lithium chloride $(3 \mathrm{mEq} / \mathrm{kg}$ intraperitoneally) 20 hours later and observed for behavioral manifestations of SE. The animals were observed for one and a half hours. Their behavior was rated as described: 0, normal; 1 , immobilization, occasional "wet dog shakes";2, head nodding, unilateral forelimb clonus, frequent "wet dog shakes"; 3 , rearing, salivation, bilateral forelimb clonus; 4 , generalized limbic seizures with falling, running and salivation; 5, continuous generalized seizures. ${ }^{[7]}$ One hour after SE, the rats were decapitated and their brains were dissected. On the day prior to sectioning, brains were immersed overnight in perfusion buffer containing $20 \%$ sucrose solution. All animal experiments were carried out in accordance with the National Institutes of Health (NIH) guide for the care and use of laboratory animals (NIH publications 80-23), and all surgical protocols have been approved by Animal Care and Use Committee. In addition, all efforts were made to lessen animal suffering and to use only the number of animals necessary to produce reliable scientific data.

\section{Histological procedures}

Detection of cell injury was performed with immunohistochemical technique on a total of 10 rats for the EP group (pilocarpine group), 10 for the rhEpo-pilocarpine group, and 6 for the $0.9 \%$ control saline group. In order to determine erythropoietin preconditioning on hippocampus neuronal injury, animals were sacrificed at one hour after SE onset. At the abovementioned time point, animals were deeply anesthetized (thionembutal, $50 \mathrm{mg} / \mathrm{kg}$, i.p.) and were perfused through the left ventricle according to the procedures of Gallyas et al. (1990, 1993) and van den Pol and Gallyas (1990). Perfusion procedures took into account the techniques developed by Cammermeyer $(1961,1967,1978)$ to avoid "dark" cell artifacts. Details on this procedure have been previously reported (Mello and Covolan, 1996). In brief, under deep anesthesia, the animals had their chests opened and the perfusion through the left ventricle of the heart was started within less than $1 \mathrm{~min}$ from chest opening, with heart frequency still above 100/min. The perfusion buffer was used for only $2 \mathrm{~min}$, and was then followed by the perfusion fixative. The buffer solution contained $0.1 \%$ $(\mathrm{w} / \mathrm{v})$ Tris, $0.59 \%(\mathrm{w} / \mathrm{v})$ calcium chloride, and $0.09 \%(\mathrm{w} / \mathrm{v})$ sodium chloride. Composition of perfusion fixative was $2 \%(\mathrm{v} /$ v) paraformaldehyde, $2.5 \%(\mathrm{v} / \mathrm{v})$ glutaraldehyde, $0.1 \%(\mathrm{w} / \mathrm{v})$ Tris, $0.59 \%$ (w/v) calcium chloride, and $0.36 \%$ (w/v) sodium chloride, with the final solution $\mathrm{pH}$ adjusted to 7.5. Removal of brains from the skull did not take place at the end of perfusion but only 24 h later, in order to avoid "dark" cell artifacts (see above). This procedure is an essential and effective measure in avoiding the staining of neurons that might occur simply due to mechanical strain to the brain if it were to be removed from skull right after perfusion ${ }^{[8]}$

\section{Caspase-3 Immunocytochemistry}

At the end of perfusion, brains were removed from the skull. They were postfixed in the same solution for 2 hours, placed in Tris phosphate-buffered saline (PBS)/30\% sucrose $\left(\mathrm{pH} 7.4,4^{\circ} \mathrm{C}\right)$ for 24 hours, and serially sectioned in 30-mm thick coronal sections on a cryotome. They were then blocked in Tris-PBS containing $3 \%$ normal goat serum (NGS) and $0.3 \%$ Triton X100 for 1 hour, incubated in the rabbit anti-caspase-3 serum (Sigma, St. Louis, MO) at a concentration of 1:1000 in 3\% NGS and $0.3 \%$ Triton X-100 overnight at room temperature, washed with Tris-PBS three times for 10 minutes each time, incubated in biotinylated goat anti-rabbit IgG (Sigma, St. Louis, MO) at 1:500 in $1 \%$ NGS and $0.3 \%$ Triton X-100 for 1 hour at room temperature, washed with Tris-PBS three times for 10 minutes each time, and then incubated in avidinbiotin-peroxidase complex (Sigma, St. Louis, MO) for 1 hour. To identify the immunoreaction product, the horseradish peroxidase was visualized with diaminobenzidine (DAB) and glucose oxidase, with nickel intensification. Coronal sections approximating $-3.6 \mathrm{~mm}$ to the bregma were used for caspase-3 expression in the Hippocampus. Both sides of several sections at each level were evaluated to ensure that the regions were truly representative. ${ }^{[9]}$ Negative control sections for each antigen were processed in the same manner except that the primary antibody incubation step was replaced by continued incubation in the normal serum. Neither immunostaining nor any recognizable background staining was observed under these conditions in negative control sections. Thus, the staining methods employed in this study clearly minimized any possible effects of the staining protocol on the visualization of caspase-3 immunoreactivity in different groups of animals. Every $30 \mathrm{~mm}$-thick section cut from the fixed slice was mounted, the number of dentate gyrus(DG), the CA1 and CA3 regions neurons were directly counted in every fifth section of the series, applying the dissector method [Figure 1]. ${ }^{[10]}$ Immunofluorescence and confocal microscopy.

Free-floating coronal sections from the whole brain were used. Sections were then incubated in a cocktail solution containing the primary antibody to caspase-3 (rabbit monoclonal, 1:200, Sigma, St. Louis, MO) and NeuN (mouse monoclonal, 1:200, Chemicon), sections were incubated for $2 \mathrm{~h}$ in one of the following secondary antibodies: goat anti-mouse conjugated to fluorescein isothiocyanate (FITC, 1:200, Sigma) and donkey anti-rabbit conjugated to Cy3 (1:300, Sigma). After washes in KPBS, sections were mounted on gelatinized slides in anti-fade medium and coverslipped. Immunofluorescent images were obtained under a confocal laser scanning microscope (Olympus FV300) and processed using Adobe Photoshop v5.0.2. 


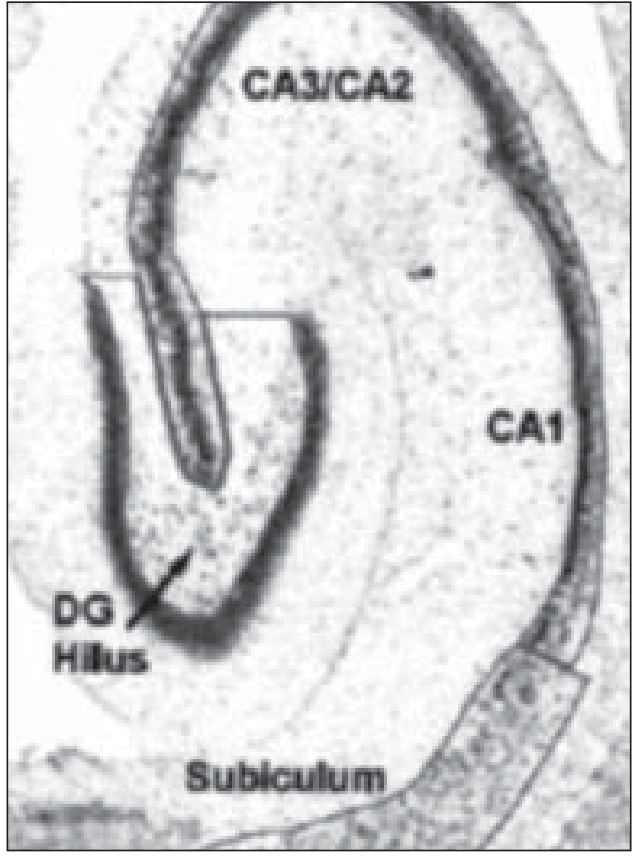

Figure 1: Boundaries of the cellular layers in hippocampus as outlined

\section{Cell quantification}

All analyses were performed by an observer blind to the treatment conditions. Immunostaining was examined with an Olympus AX70 light microscope. The number of positive cells was counted in the GCL (granule cell layer) and within two cell diameters below this region in the SGZ (referred to as GCL/SGZ) as well as in the dentate hilus. Caspase-3 staining was also assessed in the CA1 and CA3 regions. In order to reduce counting bias, only central cell profiles (Coggeshall and Lekan, 1996) exceeding $3 \mathrm{~mm}$ were included. ${ }^{[11]}$ With each staining, the number of labelled cells in three coronal sections from each rat $(5 \pm 7$ sections for the Caspase-3 counts), located between 3.3 and $4.3 \mathrm{~mm}$ posterior to bregma (encompassing the dorsal hippocampal region), was counted and expressed as average number of cells per section.

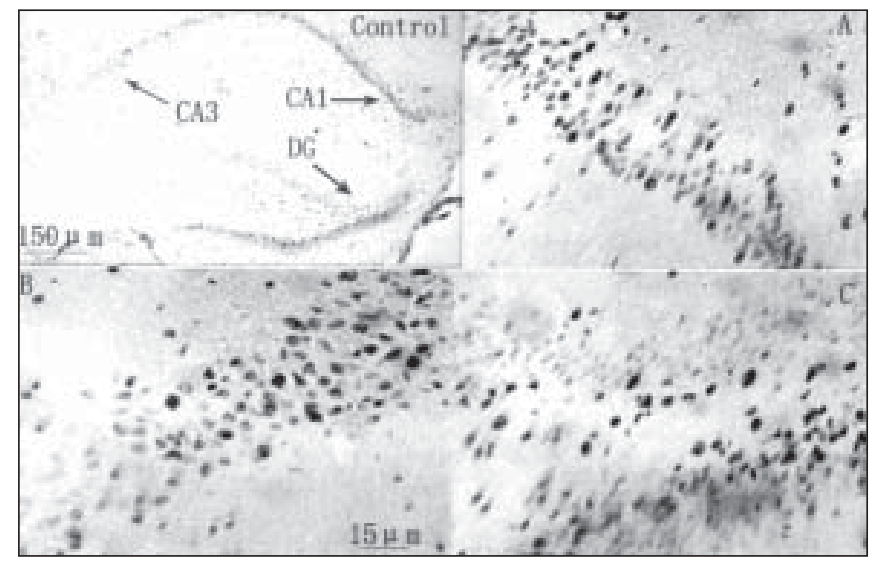

Figure 2: Caspase-3 positive cells in CA1/CA3 subfield and DG of hippocampus in control group. Figure A: CA1; Figure B: CA3; Figure C: DG. Magnification $400 \times$. Scale bar $15 \mu \mathrm{m}$. Untitled figure: hippocampus. Magnification $40 \times$. Scale bar $150 \mu \mathrm{m}$
Furthermore, the counts are reported as mean number of cells per section in one hemisphere. All cell counts were performed under $400 \mathrm{x}$ magnification.

\section{Statistical analysis}

All values are expressed as mean \pm SEM. For comparison between three treatments groups, Differences among means were determined by one-way analysis of variance (ANOVA) followed by Fisher's post hoc test. $P$ values $<0.05$ were considered significant.

\section{Results}

\section{Behavioral activity of animals after pilocarpine administration}

Behavioral activity of animals belonging to all three groups was monitored for one and a half hours after intraperitonial administration of pilocarpine. During this period, all of the pilocarpine group showed behavioral changes indicative of seizure activity, such as stage 4 or stage 5 . Seven rats $(70 \%)$ in the rhEpopilocarpine group exhibited seizure activity less than stage 4 . None of the control group showed seizure activity.

\section{Caspase-3 is expressed in hippocampus following SE}

To study the levels of the cysteine proteases family proteins in hippocampus after pilocarpine or rhEpo, we used immunohistology analyses. The pro-apoptotic protein caspase-3 is normally expressed at a low level in hippocampus [Figure 2]. However, there was a robust increase in caspase-3 immunoreactivity in cells in the dentate gyrus (DG) and CA1/ CA3 area of hippocampus at one hour of SE of pilocarpine administration [Figure 3]. Conversely, after preconditioning with rhEpo, there was a mild decrease in caspase-3 immunoreactivity in cells in the dentate gyrus (DG) and CA1/CA3 area of hippocampus at one hour of SE of pilocarpine administration

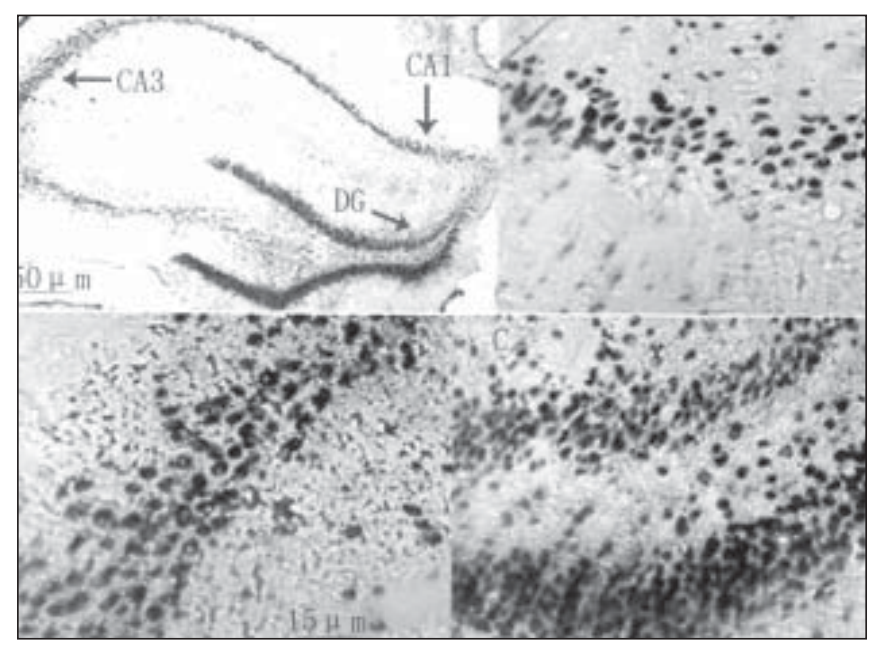

Figure 3: Caspase-3 positive cells in CA1/CA3 subfield and DG of hippocampus in EP group(pilocarpine group). Figure A: CA1; Figure B: CA3; Figure C: DG. Magnification 400x. Scale bar $15 \mu \mathrm{m}$. Untitled figure: hippocampus. Magnification 40x. Scale bar $150 \mu \mathrm{m}$ 
[Figure 4]. Quatification of hippocampus neurons revealed that the number of caspase-3-positive cells in the CA1/CA3 area $(190.55 \pm 18.74)$ and DG (318.26 \pm 37.61$)$ of vehicle - treated group had an obviously significant difference $(P=0.0281$, $p=0.0021$ ), compared with those in rhEpo-treated group (CA1/ CA3 area 270.63 \pm 19.89; DG1500.39 \pm 58.92) and EP group (CA1/CA3 area 330.79 \pm 20.46 ; DG2016.85 \pm 70.33. Both rhEpo-treated group and EP group in the CA1/CA3 area and DG had a significant difference too $(P=0.0482, P=0.0367)$. Moreover, in comparison with control group, there was an increase by $74 \%$ and $534 \%, 42 \%$ and $272 \%$ in the CA1/CA3 area and DG of EP group and rhEpo-treated group respectively. There was a decrease by $18 \%$ and $26 \%$ in the CA1/CA3 area and DG of rhEpo-treated group compared with those in EP group. [Figure 5]. Colocalization of caspase-3 with $\mathrm{NeuN}$

Brain sections were stained for NeuN (green) and caspase-3 (red). Colocalization of caspase- 3 with $\mathrm{NeuN}$ was shown by yellow nuclei [Figure 6].

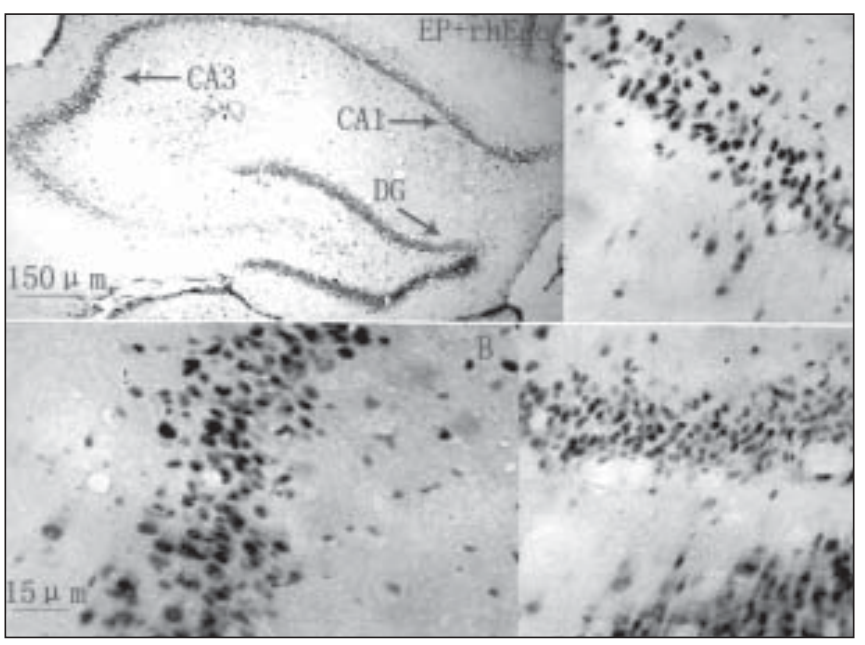

Figure 4: Caspase-3 positive cells in CA1/CA3 subfield and DG of hippocampus in rhEpo-EP group(rhEpo-pilocarpine group). Figure $A$ CA1; Figure B: CA3; Figure C: DG. Magnification 400x. Scale bar $15 \mu \mathrm{m}$ . Untitled figure: hippocampus. Magnification $40 \times$. Scale bar $150 \mu \mathrm{m}$

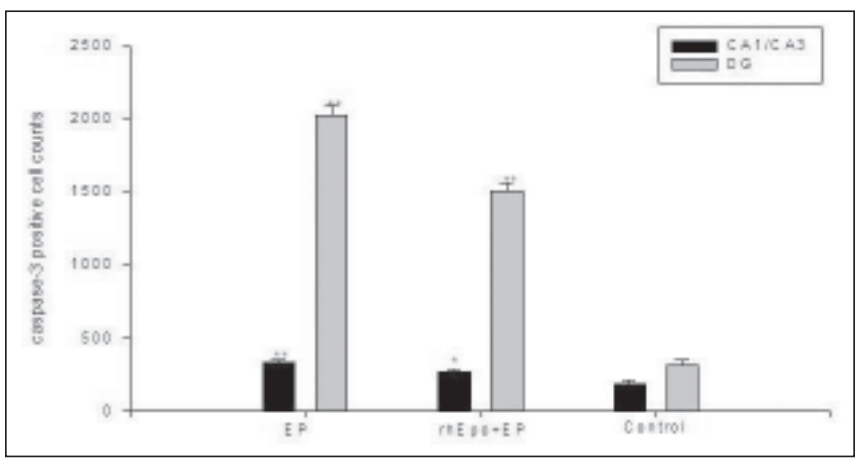

Figure 5: Caspase-3 positive cells in CA1/CA3 subfield and DG (dentate gyrus) of hippocampus in three groups. The caspase-3 positive cells were quantified one hour after SE. The regional differences are depicted, showing the average positive cells \pm SEM in the CA1/CA3 area and DG of hippocampus. ${ }^{*} P<0.05$, ${ }^{\star \star} P<0.01$, using ANOVA and Fisher's post hoc test

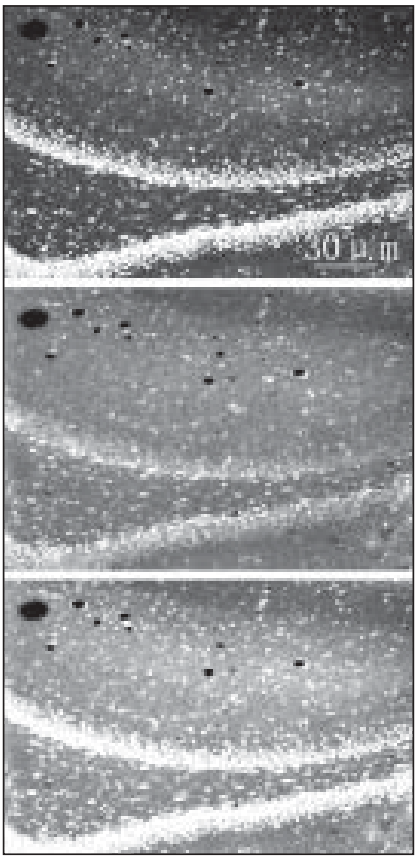

Figure 6: Colocalization of Caspase-3 with NeuN in DG of EP group Brain sections were stained for NeuN (green) and caspase-3 (red). Colocalization of caspase-3 with NeuN in DG of EP group is shown by yellow nuclei. Magnification $200 \times$. Scale bar $30 \mu \mathrm{m}$

\section{Discussion}

Our results revealed several new aspects of Epo therapy that might be useful in the management of SE. We demonstrated for the first time that Epo protects hippocampus neurons in vivo from apoptosis after SE, and that anti-apoptotic signaling pathways initiated by Epo included caspase-3 activation in hippocampal neurons.

Hypoxic-ischaemic brain injury is the major established cause of mortality and neurological morbidity in the term infant. ${ }^{[13,14]}$ Depending on the extent and the location of the insult, survivors develop permanent neurological dysfunctions such as cerebral palsy, epilepsy and mental retardation. ${ }^{[15,16]} \mathrm{SE}$ is a neurologic emergency associated with high mortality and long-term disability. ${ }^{[17,18]} \mathrm{SE}$ in humans and animal models results in significant cerebral damage and increases the risk of subsequent seizures, along with a characteristic pattern of neuronal cell loss preferentially in the hippocampus. ${ }^{[18-20]}$ Selective neuronal loss following SE was assumed to arise through hypoxia/ischemia. Experimental modeling of such brain injury suggests that seizures elicit a mixture of necrotic and apoptotic cell death within vulnerable fields. ${ }^{[5]}$ Pilocarpine-induced seizure models have provided information on the behavioral and neurochemical characteristics associated with seizure activity. This model can be used to investigate the development of neuropathology in SE. So hypoxic-ischaemic brain injury leads to neuronal apoptosis, which is involved in epileptogenesis.

During postnatal life, apoptosis plays a role in controlling the number of cells and in involution of some tissues. Apoptosis also 
can mediate cell death during acute and slowly progressing neurologic diseases. ${ }^{[21]} \mathrm{A}$ common end point of the apoptotic pathway in neurologic diseases is the activation of caspases that initiate the proteolytic mechanism involved in programmed neuronal death. ${ }^{[22]}$ Caspase-3 seems to be specifically involved in neuronal apoptosis. ${ }^{[23]}$ In the developmental brain, the ability of cytochrome c to induce activation of caspase-3 is decreased during maturation of the rat brain and is undetectable by the caspase activity assay and by Western blot in rat brain samples after 2weeks of age, a period when the brain growth spurt is levelling out. Again, activation of caspase-3 in neurons was reported to be reduced from $92.6 \%$ and $92 \%$ in $\mathrm{P} 7$ rat pups to: $65 \%$ and $38 \%$, $27 \%$ and $2 \%$, and $4 \%$ and $0.8 \%$ in $\mathrm{P} 15, \mathrm{P} 26$, and $\mathrm{P} 60$ rats, respectively, in the striatum and cortex after $\mathrm{HI}$ and $48 \mathrm{~h}$ of recovery. ${ }^{[24]}$ These data suggest a plausible mechanism to explain the shifting of pathogenesis from caspase-3-mediated neuronal death in immature neurons to non-caspase-3-mediated neuronal death in mature neurons after brain injury. However, in our experiment, in order to decrease mortality rate of rats, undergoing SE for an hour, we selected more than 2-week-old Sprague-Dawley rats. Consequently, our results were not in accordance with previous reports demonstrating caspase-3-mediated neuronal death in immature neurons rather than in mature neurons after brain injury. Because there was a robust increase in caspase-3 immunoreactivity in cells in the dentate gyrus(DG) and CA1/ CA3 area of hippocampus at one hour of SE of pilocarpine administration in our study. Although caspase-3 was suggested to play a minor role in cell death in mature neurons, active caspase3 was soon (1-3h) evident after focal stroke in the adult rat. ${ }^{[25-29]}$

In the last years, evidence has accumulated showing that the renal cytokine Epo displays a number of biological actions within the CNS that are not related to its effect on erythropoiesis. Both Epo and its receptor (Epo-R) were shown to be expressed in neurons and glial cells of human or rodent brains (Masuda et al., 1994; Nagai et al., 2001). ${ }^{[30]}$ Epo has also been shown to cross the blood-brain barrier via a receptor-mediated mechanism, ${ }^{[31]}$ indicating the potential for a direct central effect. Findings presented in this study suggest that Epo reduced neuronal apoptosis in vivo through a mechanism involving the inhibition of caspase-3 activation. After preconditioning with rhEpo, there was a mild decrease in caspase-3 immunoreactivity in cells in DG (from $2016.85 \pm 70.33$ to $1500.39 \pm 58.92$ ) and CA1/CA3 area(from $330.79 \pm 20.46$ to $270.63 \pm 19.89$ ) of hippocampus at one hour of SE of pilocarpine administration. Interestingly, $70 \%$ rats in the rhEpo-pilocarpine group exhibited seizure activity less than stage 4 . These results suggests that rhEpo could be an effective therapy for the management of SE via a specific neuroprotective mechanism.

Though we have demonstrated that systemic administration of rhEpo reduced the apoptosis of hippocampal neurons through anti-caspase-3 expression, many questions need further investigation. For example, Is Epo anticonvulsive? Are antiapoptotic genes such as bcl2/xL involved in neuroprotective mechanism of Epo? So we would explore a chronic epilepsy model in the future.

\section{References}

1. Gluck MR, Jayatilleke E, Shaw S, Rowan A.J, Haroutunian V. CNS oxidative stress associated with the kainic acid rodent model of experimental epilepsy. Epilepsy Res 2000;39:63-71.

2. Meldrum BS. Concept of activity-induced cell death in epilepsy:historical and contemporary perspectives. Prog Brain Res 2002;135:3-11.

3. Campana WM, Myers RR. Exogenous erythropoietin protects against dorsal root ganglion apoptosis and pain following peripheral nerve injury. Eur J Neurosei 2003;18:1497-506.

4. Yoon W.J, Won S.J, Ryu BR, Gwag B.J. Blockade of ionotropic glutamate receptors produces neuronal apoptosis through the Bax-cytochrome $\mathrm{C}$-caspase pathway:the causative role of $\mathrm{Ca}^{2+}$ deficiency. J Neurochem 2003;85:525-33.

5. Araki T, Simon RP, Taki W, Lan JQ, Henshall DC. Characterization of Neuronal Death Induced by Focally Evoked Limbic Seizures in the C57BL/6 Mouse. J Neurosei Res 2002;69:614-21.

6. Wang X, Zhu C, Wang X, Gerwien JG, Schrattenholz A, Sandberg M, et al. The nonerythropoietic asialoerythropoietin protects against neonatal hypoxia-ischemia as potently as erythropoietin. .J Neurochem 2004;91:900-10.

7. Racine RJ. Modification of seizure activity by electricalstimulation: a!. Motor seizures Electrorencephalogr Clin Neurophysiol 1972;32:281-94

8. Covolan L, Ribeiro LT, Longo BM, Mello LE. Cell Damage and Neurogenesis in the Dentate Granule Cell Layer of Adult Rats After Pilocarpine- or Kainate-Induced Status Epilepticus. Hippocampus 2000;10:169-80.

9. Sankar R, Shin D, Mazarati AM, Liu H, Katsumori H, Lezama R, et al Epileptogenesis after Status Epilepticus Reflects Age- and Model-Dependent Plasticity. Ann Neurol 2000;48:580-9.

10. Lister JP, Blatt G.J, DeBassio WA, Kemper TL, Tonkiss .J, Galler JR, et al. Effect of Prenatal Protein Malnutrition on Numbers of Neurons in the Principal Cell Layers of the Adult Rat Hippocampal Formation. Hippocampus 2005;15:393403.

11. Ekdahl CT, Mohapel P, Weber E, Bahr B, Blomgren K, Lindvall O. Caspase-mediated death of newly formed neurons in the adult rat dentate gyrus following status epilepticus. Eur J Neurosci 2002;16:1463-71.

12. Dal-Pizzol F, Klamt F, Vianna MM, Schröder N, Quevedo J, Benfato MS, et al. Lipid peroxidation in hippocampus early and late after status epilepticus induced by pilocarpine or kainic acid in Wistar rats, Neurosei Lett 2000;291:179-82.

13. Inder T, Volpe JJ. Mechanisms of perinatal brain injury. Semin Neonatol 2000;5:316.

14. Volpe J. Neurology of the newborn. In Neurology of the Newborn. Ed. J Volpe. Philadelphia: Saunders; 1995 . p. 1-876.

15. Berger R, Garnier Y. Pathophysiology of perinatal brain damage. Brain Res Rev 1999;30:107-34.

16. Mercuri E, Cowan F. Cerebral infarction in the newborn infant: review of the literature and personal experience. Eur J Paediatr Neurol 1999;3:255-63.

17. Manno EM. New management strategies in the treatment of status epilepticus. Mayo Clin Proc2003;78:508-18.

18. Wasterlain CG, Fujikawa DG, Penix L. Pathophysiological mechanism of brain damage from status epilepticus. Epilepsia 1993;34:S37-53

19. Mikati MA, Abi-Habib R.J, El Sabban ME, Dbaibo GS, Kurdi RM, Kobeissi M, et al. Hippocampal programmed cell death after status epilepticus: evidence for NMDAreceptor and ceramide-mediated mechanisms. Epilepsia 2003;44:282-91.

20. DeGiorgio CM, TRomivasu U, Gott PS. Hippocampal pyramidal cell loss in human status epilepticus. Epilepsia 1991;33:23-7.

21. DeGiorgio CM, Tomiyasu U, Gott PS, Treiman DM. Is the cell death in Mesial Temporal sclerosis apoptotic? Epilepsia 1992;33:23-7.

22. Schulz JB, Weller M, Moskowitz MA. Caspases as treatment targets in stroke and neurodegenerative disease. Annl Neurol 1999;45:421-9.

23. Faherty CJ, Xanthoudakis S, Smeyne RJ. Caspase-3 dependent neuronal death in the hippocampus following kainic acid treatrment. Mol Brain Res 1999;70:159-63.

24. Benjelloun N, Joly LM, Palmier B, Plotkine M, Charriaut-Marlangue C. Apoptotic mitochondrial pathway in neurons and astrocytes after neonatal hypoxia-ischaemia in the rat brain. Neuropathol Appl Neurobiol 2003;29:350-60.

25. Benchoua A, Guegan C, Couriaud C, Hosseini H, Sampaio N, Morin D, et al. Specific caspase pathways are activated in the two stages of cerebral infaretion. J Neurosei 2001;21:7127-34

26. Chen .J, Nagayama T, .Jin K, Stetler R, Zhu R, Graham S, Simon R. Induction of caspase-3 like protease may mediate delayed neuronal death in the hippocampus after transient cerebral ischemia. J Neurosei 1998;18:4914-28.

27. Krupinski J, Lopez E, Marti E, Ferrer I. Expression of caspases and their substrates in the rat model of focal ischemia. Neurobiol Dis 2000;7:332-42.

28. Namura S, Zhu J, Fink K, Endres M, Srinivasan A, Tomaselli K.J, et al. Activation and cleavage of caspase-3 in apoptosis induced by experimental cerebral ischemia. 
J Neurosei 1998;18:3659-68.

29. Velier JJ, Ellison JA, Kikly KK, Spera PA, Barone FC, Feuerstein GZ. Caspase8 and caspase- 3 are expressed by different populations of cortical neurons undergoing delayed cell death after focal stroke in the rat. .J Neurosci 1999;19:5932-41.

30. Vairano M, Dello Russo C, Pozzoli G, Battaglia A, Scambia G, Tringali G, et al

\section{Invited Comments}

The accompanying article titled 'Erythropoietin preconditioning' on hippocampus neuronal apoptosis following status epilepticus induced by Li-pilocarpine in rats through anti-caspase-3 expression' examines the neuroprotective role of erythropoietin (EPO) in epilepsy. In recent years, EPO and its receptor (EPOR) have been shown to be present in the nervous system and EPO is neuroprotective in models of a variety of neurological illnesses. These range from acute illnesses such as stroke and spinal cord injury to more chronic illnesses such as peripheral neuropathies. ${ }^{[1,2]}$ A Phase II clinical trial of EPO has been completed ${ }^{[3]}$ and a larger multi-center Phase III trial of EPO in stroke is underway in Germany. The article by Wen and colleagues is the first demonstration that EPO may be of benefit in epilepsy. They use a standard animal model of seizure induction in rats and show that pre-treatment of the animals with EPO partially prevents development of seizures and seizure induced neuronal death in this model. However, this is probably the first step in a line of investigation to evaluate a potential therapeutic role for EPO in epilepsy. In most neuroprotective animal models, the investigators administer the neuroprotective drug first before the insult and then after the insult to mimic what would happen in patients.
Erythropoietin exerts anti-apoptotic effects on rat microglial cells in vitro. Eur J Neurosei 2002;16:584-92.

31. Brines ML, Ghezzi P, Keenan S, Agnello D, de Lanerolle NC, Cerami C, et al Erythropoietin crosses the blood-brain barrier to protect against experimental brain injury. Proc Natl Acad Sci U S A 2000;97:10526-31.

Accepted on 03-12-2005

Unfortunately, this second step was not done in this study and will need to be done before one can be more confident of a potential therapeutic role for EPO in epilepsy. Furthermore, in most patients seizures are part of a chronic epileptic illness and potential utility of EPO needs to be tested in an animal model of chronic epilepsy. Having said that though, recombinant human EPO is relatively safe and can be used in the context of a clinical trial in status epilepticus patients if animal studies show benefit when EPO is given after the onset of seizure activity.

Ahmet Hoke

Department of Neurology, Johns Hopkins University, School of Medicine Path 509, 600 N. Wolfe St. Baltimore, MD, 21287, USA E-mail: ahoke@jhmi.edu

\section{References}

1. Höke A, editor. Erythropoietin and the Nervous System. Springer: Boston; 2006. p. 1-223.

2. Lipton SA. Erythropoietin for neurologic protection and diabetic neuropathy. $\mathrm{N}$ Eng J Med 2004;350:2516-7.

3. Ehrenreich H, Hasselblatt M, Dembowski C, Cepek L, Lewczuk P, Stiefel M, et al. Erythropoietin therapy for acute stroke is both safe and beneficial. Mol Med $2002 ; 8: 495-505$. 